\title{
Plasma carcinoembryonic antigen concentrations and immunohistochemical patterns of epithelial marker antigens in patients with large bowel carcinoma
}

\author{
TO ROGNUM, K ELGJO, P BRANDTZAEG, H ØRJASAETER, A BERGAN
}

From the Histochemical Laboratory, Institute of Pathology and Surgical Department B, the National Hospital, Rikshospitalet, and the Department of Immunology, National Institute of Public Health, Oslo, Norway

SUMMARY Carcinoembryonic antigen (CEA), secretory component (SC), and epithelial IgA were traced by paired immunofluorescence staining in 102 large bowel carcinomas from 99 patients. The immunohistochemical results were evaluated semiquantitatively in relation to histological tumour grade, clinicopathological stage, and preoperative plasma CEA concentration. CEA expression was significantly increased $(p<0.05)$ in the following order: histologically normal colon mucosa, transitional mucosa adjacent to tumours, neoplastic epithelium; the reverse was true for the expression of SC and epithelial IgA $(p<0 \cdot 01)$. CEA was significantly more abundant in the moderately and poorly differentiated tumours than in the well differentiated ones $(p<$ $0.05)$, whereas the latter showed better expression of $\mathrm{SC}(\mathrm{p}<0.05)$ and epithelial $\operatorname{IgA}(\mathrm{p}$ $\Omega 0.06)$. In the transitional mucosa, CEA staining tended to be inversely related to histological tumour grade, whereas SC and epithelial $\operatorname{IgA}$ were significantly better seen in this zone when the adjacent tumour was well differentiated than when it was moderately or poorly differentiated (ps $<0.01)$. Furthermore, the expression of SC and epithelial $\operatorname{IgA}$ in the transitional mucosa decreased with increasing invasiveness of the tumours, whereas the opposite relation was indicated for CEA expression. Plasma CEA concentrations were not clearly correlated with histological tumour grade, but the localised well differentiated tumours tended to be associated with lower levels than the localised moderately differentiated ones $(\mathrm{p} \bumpeq 0.06)$. Moreover, the latter variety was associated with lower plasma CEA concentrations than disseminated tumours of comparable differentiation $(\mathrm{p}<0 \cdot 01)$.

The malignant potential of large bowel carcinomas has up till now been predicted on the basis of pathological staging and histopathological grading.' In addition, pre- and postoperative measurements of carcinoembryonic antigen (CEA) in plasma may aid monitoring of cancer treatment. ${ }^{2}$ Recently, expression of epithelial marker antigens has been suggested as a useful tumour characteristic. $\mathrm{CEA}^{3}$ and components of the secretory $\operatorname{IgA}$ system $^{4}$ have thus been studied in gastric malignancies ${ }^{5}$ and in large bowel carcinomas. ${ }^{6}$ The latter investigation was based on semiquantitative and concomitant evaluation of CEA, secretory component (SC), and epithelial IgA by paired immunofluorescence staining. Although the cases were relatively few and the individual variations large, the expression of SC and

Accepted for publication 2 February 1982
IgA in neoplastic epithelium was, on the whole, positively correlated with the histological degree of tumour differentiation. Conversely, there was no apparent relation between expression of CEA and tumour differentiation.

In this extended study we evaluated immunohistochemically the same three epithelial markers in 102 large bowel carcinomas in relation to histological tumour grade and clinicopathological stage. The preoperative plasma CEA levels of the 99 included patients were also taken into account.

\section{Material and methods}

TISSUE SPECIMENS

Altogether 102 tumour specimens were obtained from 99 patients ( 47 men and 52 women) who were subjected to surgery for adenocarcinoma of the 
large bowel. Their mean age was $63 \mathrm{yr}$ (range: 28-90 yr).

Immediately after resection, the tissue material was transported in ice-cold phosphate-buffered saline (PBS) $(0.01 M$ phosphate buffer, $\mathrm{pH} 7 \cdot 6$, containing $0.15 \mathrm{M} \mathrm{NaCl}$ ) to the laboratory where the macroscopic examination was done, and appropriate tissue samples were excised for immunohistochemistry. All samples were placed in cold $96 \%$ ethanol and processed for paraffin embedding as detailed elsewhere.

\section{HISTOPATHOLOGICAL GRADING}

Serial sections were cut at $6 \mu \mathrm{m}$. The first section in every series was stained for histopathological evaluation with a trichrome routine method combining haematoxylin, azofloxine, and saffron (HAS). ${ }^{8}$ After randomisation of these sections, the tumours were graded as poorly, moderately, or well differentiated on the basis of Evans' criteria.9 The same trained pathologist was responsible for this grading throughout the study, and a blind test for reproducibility did not reveal any systematic error. ${ }^{6}$

\section{CLINICOPATHOLOGICAL STAGING}

The extent of bowel involvement and the presence or absence of lymph node metastasis were staged in accordance with the criteria given by Dukes and Bussey.' In addition to Dukes' stages A, B, and C, tumours with distant organ metastasis were assigned stage $D$.

\section{IMMUNOHISTOCHEMISTRY}

The immunohistochemical investigation was for most tumours based on serial sections from two tissue blocks-both containing neoplastic epithelium and the adjacent transitional mucosa. As controls, 47 histologically normal samples of large bowel mucosa were included. One section of each series was incubated with fluorescein isothiocyanate (FITC)-labelled sheep anti-SC and tetramethylrhodamine isothiocyanate (MRITC)-labelled rabbit anti-CEA, and another with FITC-labelled rabbit anti-IgA and MRITC-labelled sheep anti-SC. The preparation and characterisation of these sheep and rabbit IgG-fluorochrome conjugates have been reported previously. ${ }^{\circ}$

Evaluation of paired immunofluorescence staining was performed in a Leitz Orthoplan fluorescence microscope equipped with an Osram HBO $200 \mathrm{~W}$ lamp for excitation of rhodamine (red) emission, and with an XBO $150 \mathrm{~W}$ lamp for fluorescein (green). Narrow-band excitation and selective filtration of the fluorescence colours were obtained with a Ploem-type epi-illuminator. The staining of the three epithelial marker antigens (CEA, SC, and
IgA) was scored semiquantitatively as detailed elsewhere. ${ }^{6}$ Briefly, a score of three indicated abundant staining reaction and 0 negligible or no reaction. Both the tumour and the adjacent transitional mucosa were evaluated. The same investigator was responsible for this scoring throughout the investigation, and a blind test for reproducibility did not reveal any systematic error. ${ }^{6}$

\section{PLASMA CEA LEVELS}

The concentration of CEA in plasma was quantified with a slightly modified Roche radioimmunoassay. ${ }^{10}$

\section{STATISTICAL ANAL YSES}

Since our observations were mainly recorded as scores, grades, or stages, only statistical methods compatible with the application of the ordinal scale were considered valid." Non-parametric rank methods were therefore used, and distributions were given as medians and observed ranges. Group comparisons were based on the Mann Whitney U test. ${ }^{12}$

\section{Results}

\section{HISTOLOGICAL GRADING}

Approximately $10 \%$ of the tumours were well differentiated and $20 \%$ poorly differentiated; most of them were, however, classified as moderately differentiated (Table). All well differentiated tumours were localised (Dukes' stages A or B), whereas 17 of the 22 poorly differentiated were disseminated (Table).

\section{IMMUNOFLUORESCENCE STAINING}

\section{CHARACTERISTICS}

When neoplastic epithelium contained detectable cell markers, they were generally distributed fairly evenly throughout the tumour. If only isolated positive epithelial elements were seen in an otherwise negative tumour, such areas were disregarded when fluorescence scores were assigned (Fig. 1).

Tumours that were SC-positive usually exhibited diffuse intracellular staining but apical intensification was noted in glandular elements (Figs. 1 and 2). Epithelial staining for $\operatorname{IgA}$ was generally confined to the apical aspects of the cells (Fig. 2).

Table Relation between histological tumour grade and clinicopathological stage

\begin{tabular}{lrrrrc}
\hline Tumour grade & \multicolumn{6}{c}{ Clinicopathological stage } \\
\cline { 2 - 6 } & \multicolumn{1}{c}{$\boldsymbol{A}$} & \multicolumn{1}{c}{$B$} & $C$ & $D$ & Total No \\
\hline Well differentiated & 6 & 5 & - & - & 11 \\
Moderately differentiated & 13 & 32 & 12 & 12 & 69 \\
Poorly differentiated & 2 & 3 & 10 & 7 & 22 \\
Total No & 21 & 40 & 22 & 19 & 102 \\
\hline
\end{tabular}



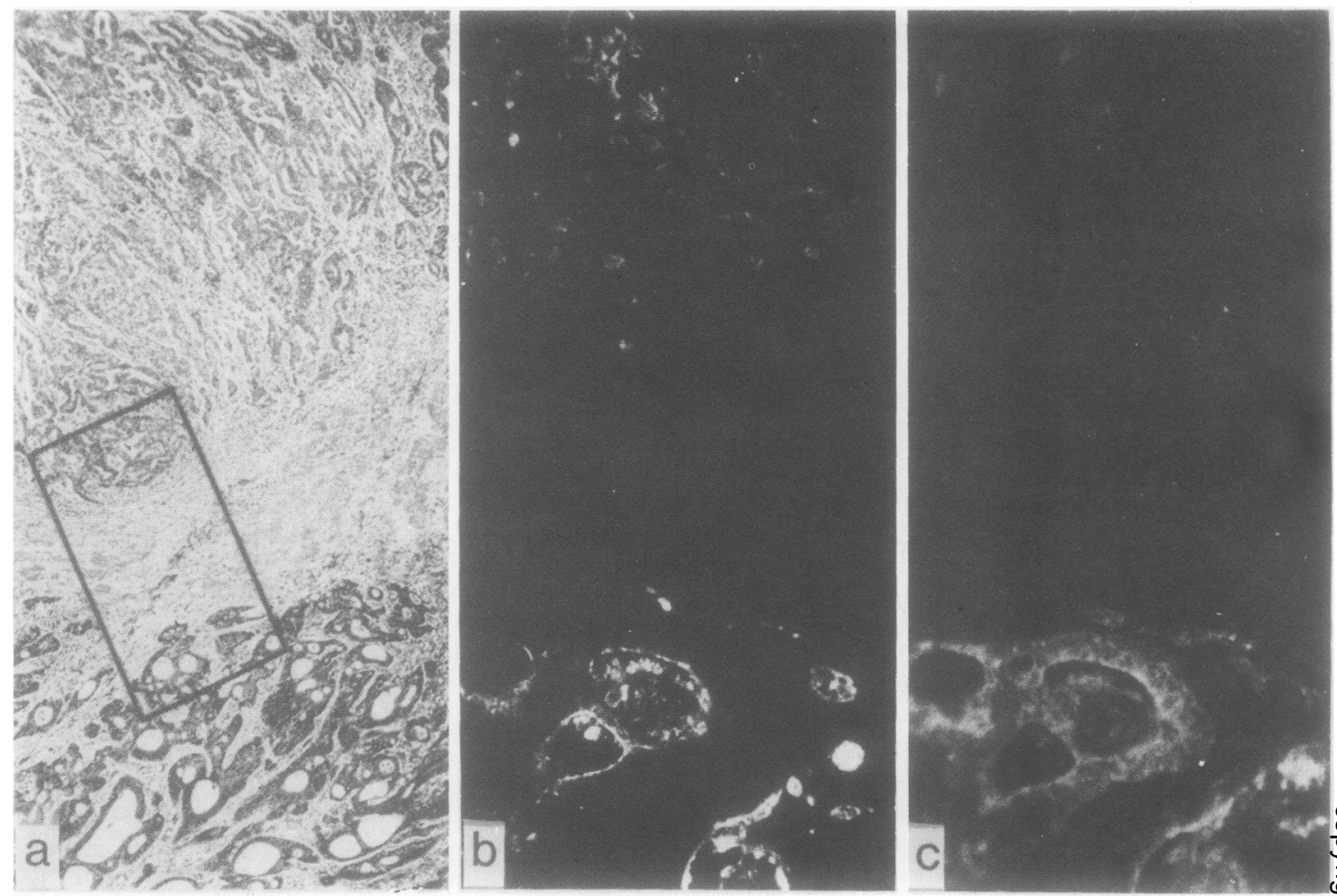

Fig. 1 Poorly differentiated adenocarcinoma with a better differentiated area in the lower part. (a) HAS-stained section. Area corresponding to frame was studied immunohistochemically in adjacent sections; $(b, c)$ same field of adjacent section stained by paired immunofuorescence for $C E A(b)$ and $S C(c)$. The better differentiated part contains both CEA and SC, whereas only traces of CEA can be seen in the undifferentiated part. $(a) \times 40,(b, c) \times 125$

Accumulations within glandular lumina were sometimes positive for both SC and IgA (Fig. 2).

Staining of CEA was principally confined to a rim lining the apical surfaces of the tumour cells (Figs. 1 and 2), but in most cases the fluorescence was intensified by intercellular extensions and often also by diffuse cytoplasmic staining (Fig. 3); CEApositive material was frequently seen in lumina of gland-forming elements (Figs. 1 and 2).

The content of goblet-like cells in well differentiated carcinomas was always negative for SC, IgA, and CEA. However, in a case of signet-ring cell carcinoma the neoplastic cells contained all three markers (Fig. 3).

In the transitional mucosa adjacent to the tumour, all three epithelial markers were distinctly localised in the columnar cells whereas the mucin content of goblet cells was unstained (Fig. 3). Some heterogeneity with regard to staining pattern was noted as reported previously; 6 each final fluorescence score was, therefore, in most cases an average based on two mucosal samples from the same specimen. When hyperplastic crypt elements were present, staining for CEA was generally intense whereas that for SC and epithelial $\operatorname{IgA}$ was faint (Fig. 4).

Four poorly differentiated carcinomas were negative for CEA-one of them being an adenoacanthoma of the caecum (Fig. 5). However, in all these cases the transitional mucosa contained CEA (Fig. $5)$.

OVERALL EXPRESSION OF EPITHELIAL MARKERS The transitional mucosa showed significantly more abundant CEA staining than histologically normal colon mucosa, and the scores given for the tumour epithelium were still higher (Fig. 6). SC and IgA produced the inverse result in relation to the three categories of epithelium (Fig. 6).

\section{EXPRESSION OF EPITHELIAL MARKERS IN} RELATION TO HISTOLOGICAL TUMOUR GRADE AND CLINICOPATHOLOGICAL STAGE

The well differentiated carcinomas were scored significantly lower for CEA than both the moderately and the poorly differentiated ones; it should be 


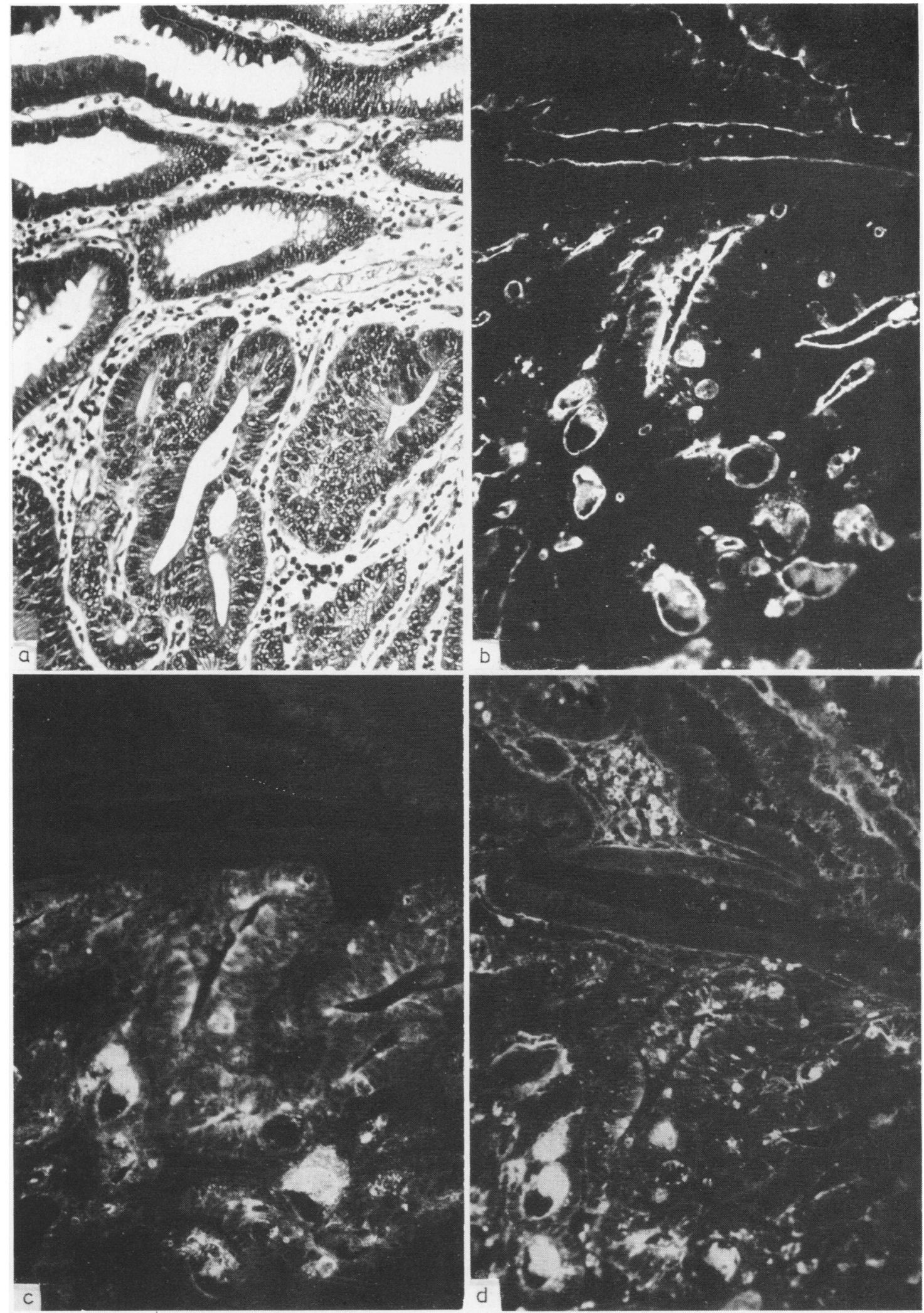

Fig. 2 Moderately differentiated adenocarcinoma in a villous adenoma; transition between villous elements (upper part) and carcinoma (lower part). (a) HAS-stained section; $(b, c)$ same field from adjacent section stained by paired immunofuorescence for CEA (b) and SC (c); (d) comparable field from a third serial section stained by immunofuorescence for IgA. The three epithelial cell markers are more strongly expressed in the carcinomatous glands than in the villous adenoma. The staining is confined to the apical part of the cells, and accumulations within the glandular lumina are positive for all three components. $\times 140$ 


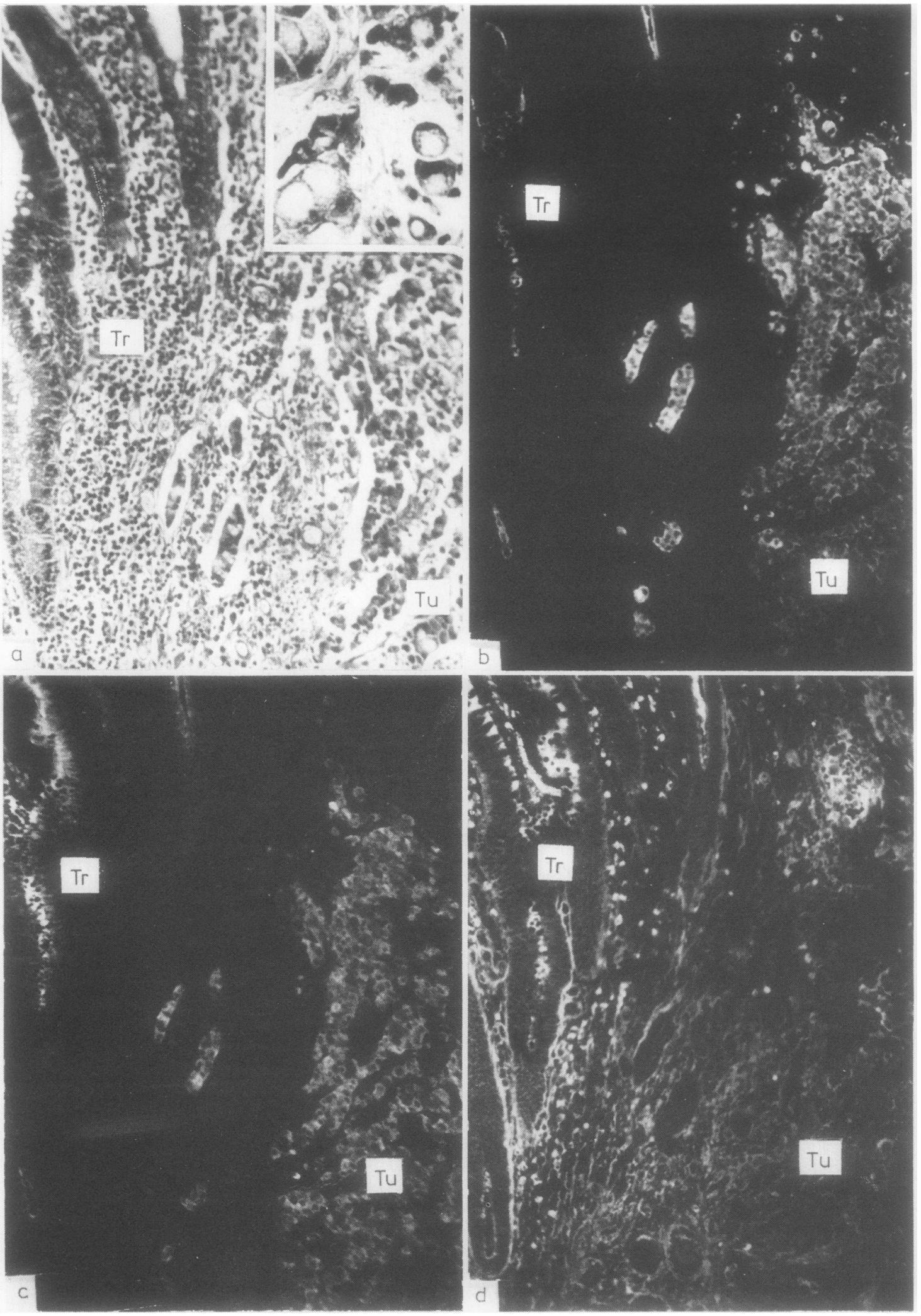

Fig. 3 . Signet-ring cell carcinoma of the caecum. (a) HAS-stained section; $(b, c)$ same field from adjacent section stained by paired immunofuorescence for CEA (b) and SC (c); (D) comparable field from a third serial section stained by immunofluorescence for IgA. In the transitional mucosa (Tr) SC and IgA are expressed by columnar epithelial cells with apical intensification, whereas CEA only occurs as a tiny luminal rim. The signet-ring cells of the tumour (Tu) are positive for CEA and SC and some of them show uptake of IgA. Numerous IgA-producing plasma cells are present in Tr. $\times 140$. Insert in $(a) \times 40$. 


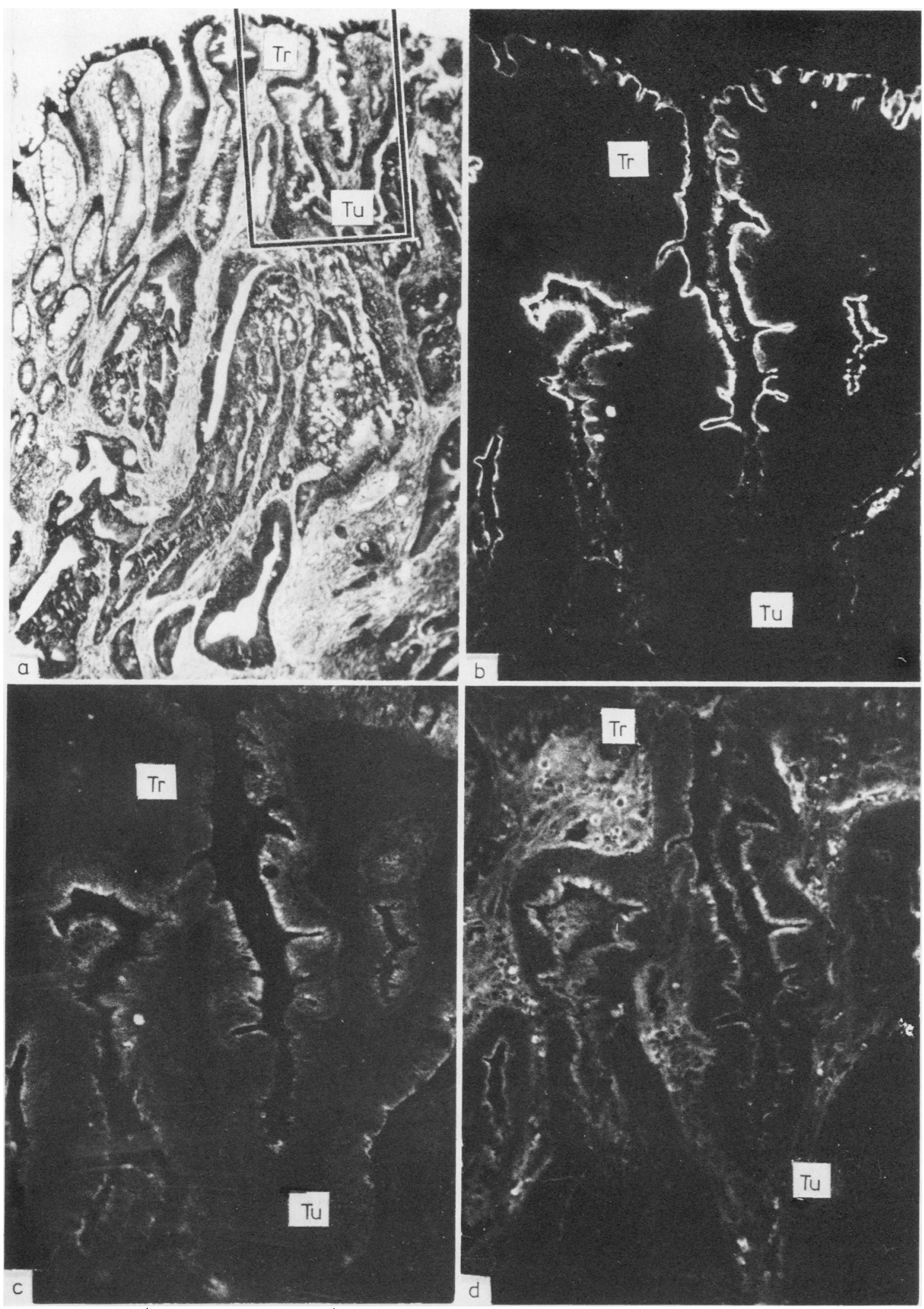

Fig. 4 Transitional mucosa (Tr) adjacent to a moderately differentiated adenocarcinoma (Tu). (a) HAS-stained section shows that the crypts adjacent to the tumour are lined by serrated hyperplastic epithelial elements. Area corresponding to frame is shown immunohistochemically in adjacent sections; $(b, c)$ same field in adjacent section stained by paired immunofuorescence for CEA (b) and SC (c); (d) comparable field in a third serial section stained by immunofuorescence for IgA. The hyperplastic epithelial elements show a broad and intense luminal border of CEA-positive material, whereas the staining for SC is faint and more diffuse. Epithelial IgA is confined to the apical part of the cells and is in some parts of the crypts almost lacking. $(a) \times 40(b, c, d) \times 140$. 


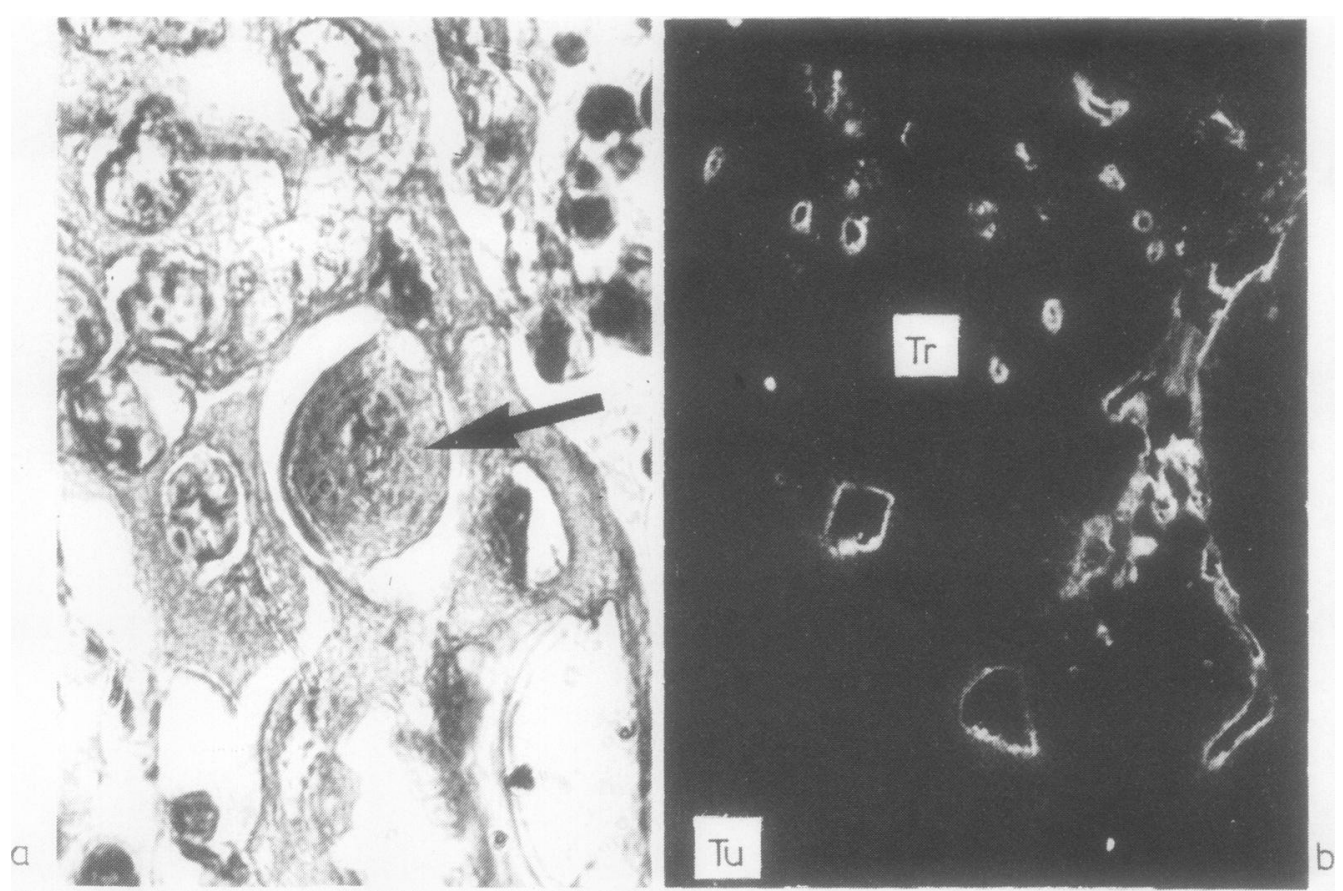

Fig. 5 Adenoacanthoma of the caecum. (a) HAS-stained section shows sheets of squamous-like cells with a central dyskeratotic one (arrow); (b) section from the same tumour specimen stained by immunofuorescence for CEA, which abounds in the transitional mucosa (Tr) but is lacking in the tumour (Tu). (a) $\times 420(b) \times 125$.

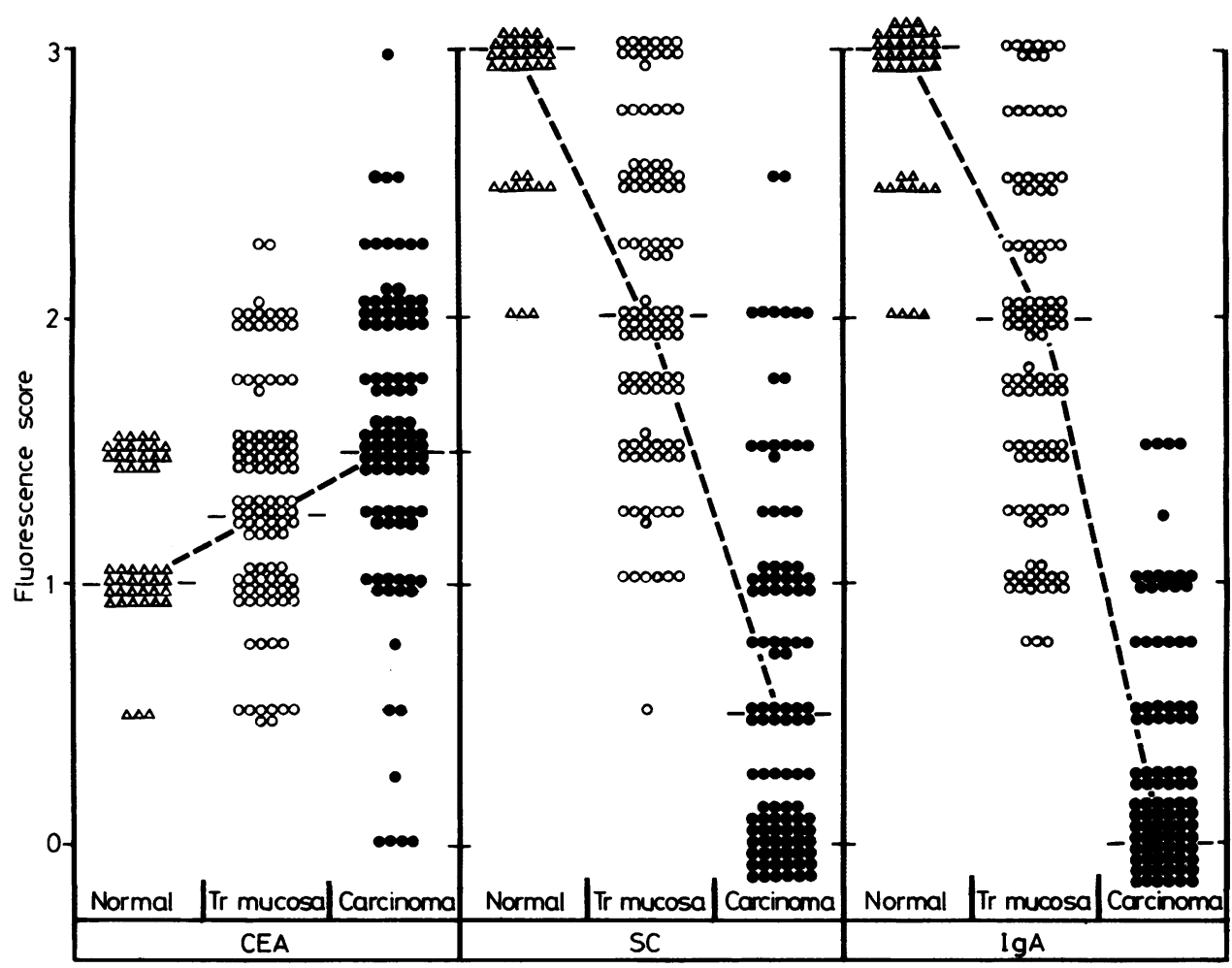

Fig. 6 Immunofuorescence scores given for epithelial staining of histologically normal colon mucosa $(\triangle)$, transitional mucosa adjacent to carcinoma (O), or carcinoma $(\bullet)$. Medians are connected by dashed lines. Transitional mucosa was scored significantly higher for CEA than normal mucosa $(p<0.05)$, and carcinoma higher than transitional mucosa $(p<0.01)$. Scores for SC-and epithelial IgA were significantly lower in transitional mucosa than in normal mucosa $(p<0.01)$, and lower in carcinoma than in transitional mucosa $(p<0.01)$. 


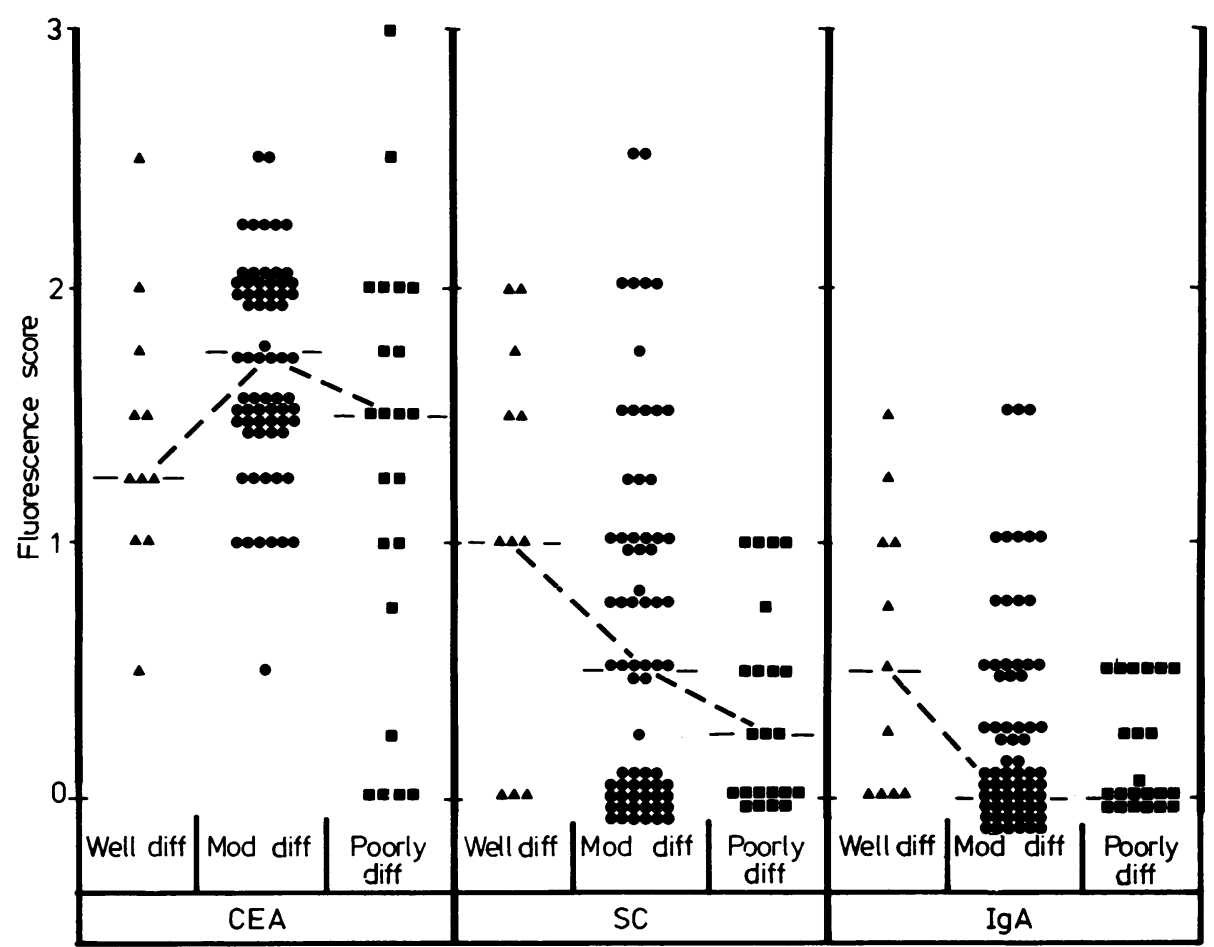

Fig. 7 Immunoftuorescence scores given for epithelial staining of poorly (৫), moderately (๑), or well differentiated $(\Delta)$, carcinomas. Medians are connected by dashed lines. Well differentiated carcinomas were scored significantly lower for CEA than moderately $(p<0.05)$ or poorly differentiated carcinomas $(p<0.05)$. Well differentiated carcinomas tended to express more $S C$ and $\operatorname{IgA}$ than moderately differentiated $(p \bumpeq 0.08$ and $p \bumpeq 0.09$, respectively), and moderately and poorly differentiated carcinomas taken together were scored significantly lower than well differentiated ones ( $p<0.05$ for $S C$ and $p=0.06$ for $\operatorname{IgA}$ ). No statistically significant differences appeared between moderately and poorly differentiated carcinomas.

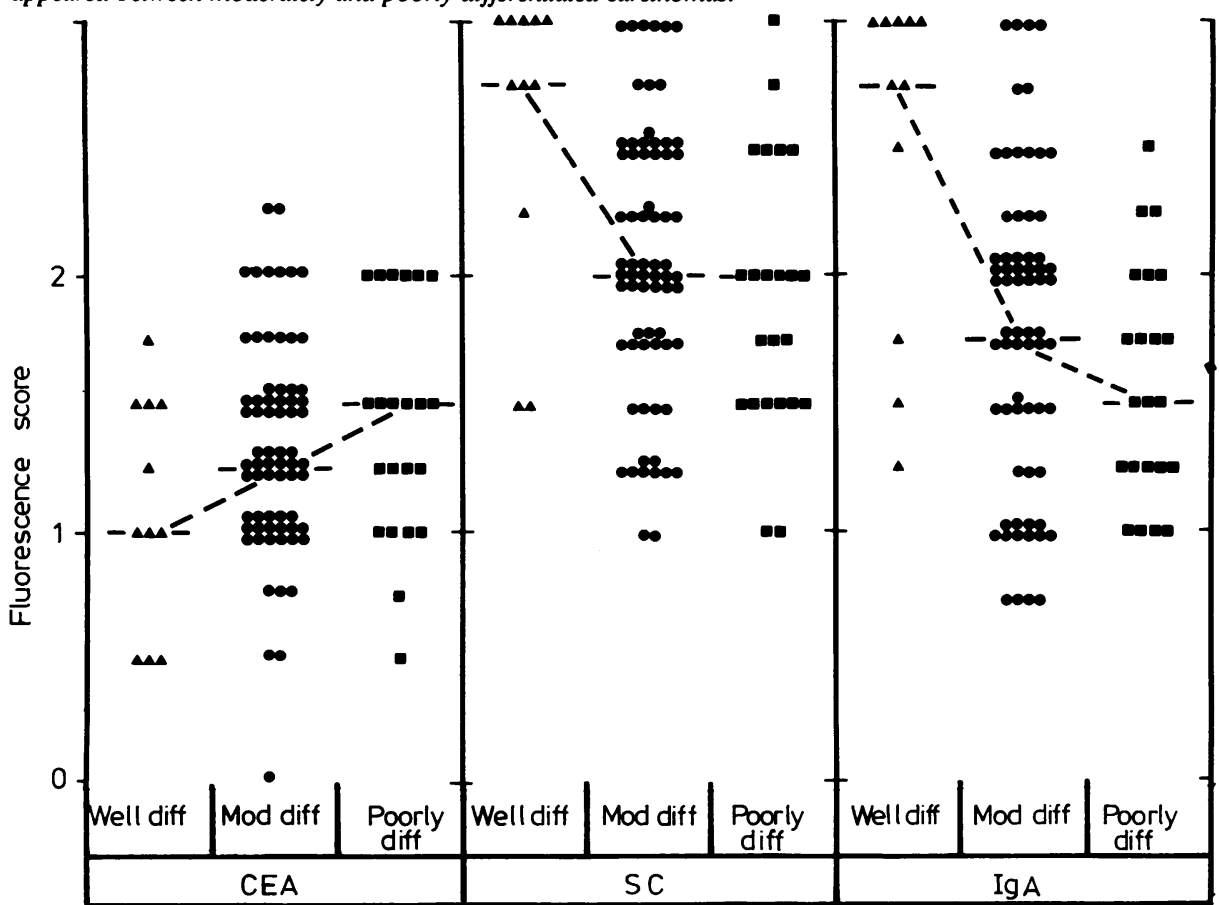

Fig. 8 Immunoftuorescence scores for epithelial staining of transitional mucosa adjacent to poorly ( $(\square)$, moderately $(\bullet)$, or well differentiated ( $\Delta$ ) carcinomas. Medians are connected by dashed lines. Transitional mucosa adjacent to well differentiated tended to express less $C E A$ than adjacent to poorly differentiated carcinomas ( $p=0.05$ ). SC and IgA scores were significantly higher adjacent to well differentiated than adjacent to moderately or poorly differentiated carcinomas $(p<0.01$ for both markers). No statistically significant differences appeared between specimens with moderately and poorly differentiated carcinomas. 


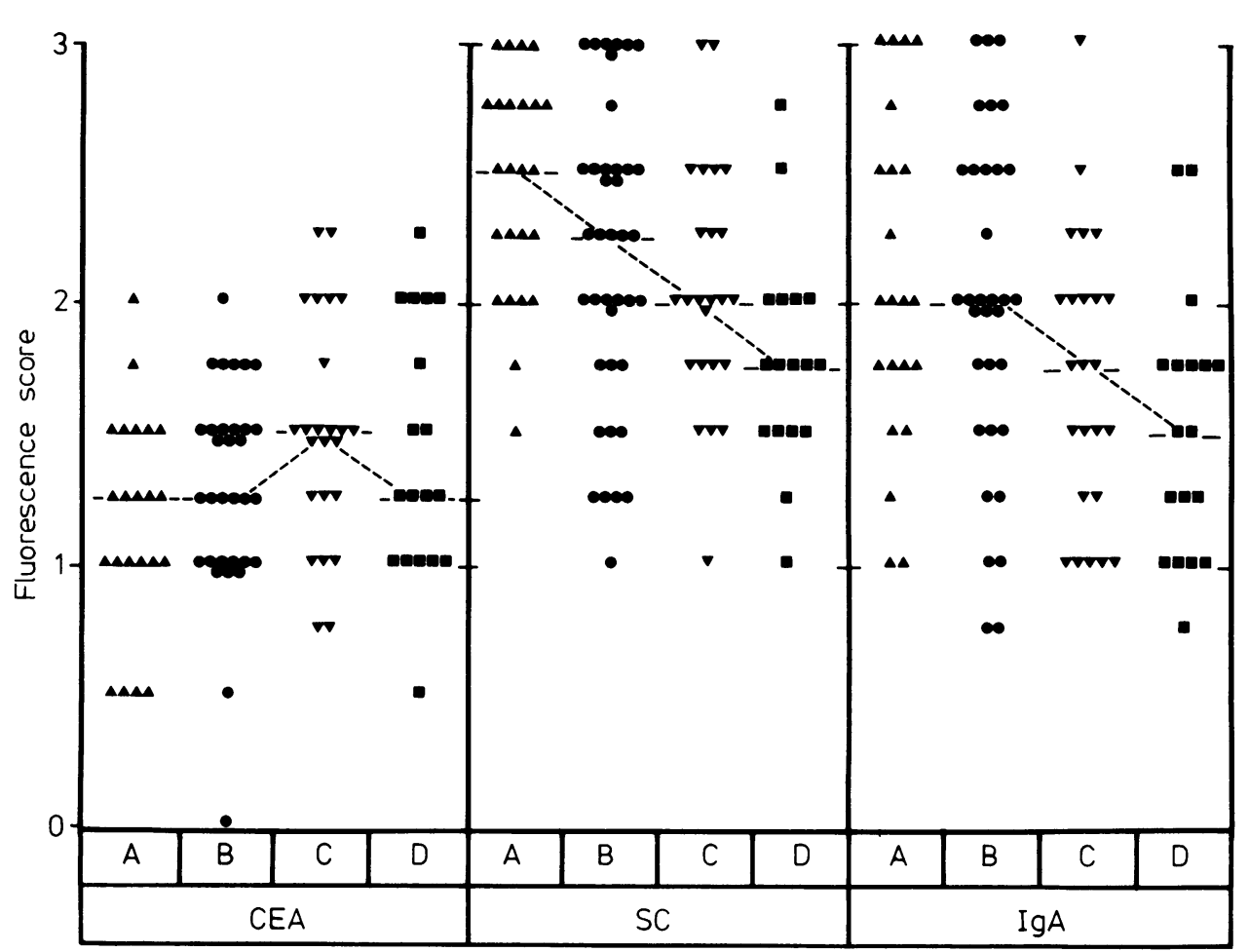

Fig. 9 Immunofluorescence scores given for epithelial staining of transitional mucosa adjacent to carcinomas of stage $A(\mathbf{\Delta}), B(\bullet), C(\nabla)$, or $D(\bullet)$. Medians are connected by dashed lines. Transitional mucosa adjacent to localised carcinoma (stages $A$ and $B$ ) tended to express less $C E A$ than adjacent to disseminated (stages $C$ and $D)(p<0.05)$, whereas expression of $S C$ and $\operatorname{Ig} A$ was best adjacent to the localised tumours $(p<0.01)$.

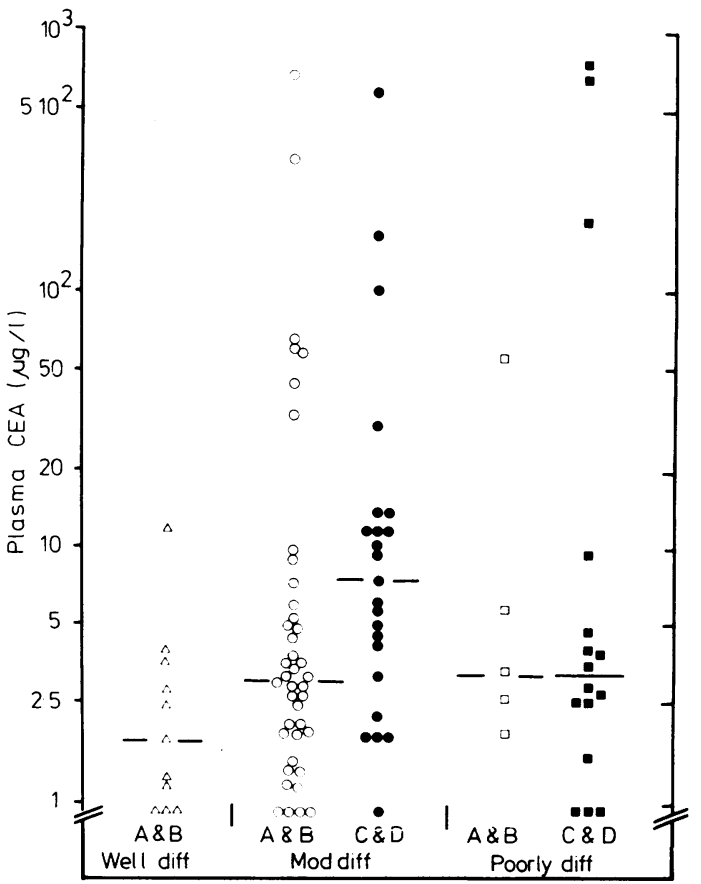

Fig. 10 Scatterdiagram of relation between histological tumour grade and plasma CEA level in patients with localised (stages $A$ and $B$ ) or disseminated adenocarcinomas (stages $C$ and $D$ ). Medians are indicated by horizontal lines. For all stages taken together, moderately differentiated carcinomas were associated with significantly higher plasma CEA concentrations than well differentiated $(p<0.02)$. The same trend was indicated after exclusion of disseminated tumours $(p \bumpeq 0 \cdot 06)$. No statistically significant differences appeared between poorly and moderately differentiated carcinomas. However, disseminated tumours were associated with significantly higher plasma $C E A$ values than localised ones only in the latter group $(p<0.05)$. 
noted, however, that among the latter there were four cases completely negative for CEA (Fig. 7). Conversely, both SC and IgA scores were significantly higher in well differentiated carcinomas than in the two other categories (Fig. 7). No statistically significant differences were found between moderately and poorly differentiated carcinomas for any of the three marker proteins (Fig. 7). Expression of CEA in neoplastic epithelium was apparently unrelated to stage, whereas SC (but less clearly $\operatorname{IgA}$ ) decreased from localised ( $A$ and $B$ ) to disseminated $(C$ and $D)$ tumours $(p<0.02)$.

The transitional mucosa tended to express more CEA adjacent to poorly differentiated than adjacent to well differentiated tumours (Fig. 8). Conversely, SC and IgA scores were significantly higher in this zone adjacent to well differentiated than adjacent to moderately or poorly differentiated carcinomas, but there was no distinct difference between the two latter varieties (Fig. 8). Expression of SC and epithelial $\operatorname{IgA}$ in the transitional mucosa was inversely related to stage whereas a positive relation was indicated for CEA expression (Fig. 9).

\section{PLASMA CEA CONCENTRATION IN RELATION TO HISTOLOGICAL TUMOUR GRADE AND \\ CLINICOPATHOLOGICAL STAGE}

Plasma CEA concentrations were not clearly correlated with histological tumour grade, and in the whole material only a weak positive correlation with stage appeared. Well differentiated carcinomas, which all belonged to stage $\mathrm{A}$ or $\mathrm{B}$, tended to be associated with lower plasma CEA concentrations than both localised and disseminated (stages $\mathrm{C}$ or D) moderately differentiated carcinomas (Fig. 10). Furthermore, among the latter the disseminated were associated with significantly higher plasma CEA concentrations than the localised ones, whereas no such difference was observed in the relatively small group of poorly differentiated carcinomas (Fig. 10).

\section{Discussion}

The proportion (about $60 \%$ ) of localised (stages A or B) adenocarcinomas of the large bowel was somewhat higher in the present material than in larger series of patients. ${ }^{13}$ Nevertheless, the distribution of different histological tumour grades was in agreement with that reported by Dukes and Bussey $;^{1}$ they found in a material of 2097 rectal carcinomas $20 \%$ poorly, $61 \%$ moderately, and $19 \%$ well differentiated tumours. Such classification of carcinomas is necessarily arbitrary with ill defined boundaries between the three histological grades. It seems important, therefore, to search for more objective criteria for prediction of malignant tumour potential. To this end we evaluated the expression of different types of epithelial cell markers both in the tumour and in its adjacent transitional mucosa.

Previous studies had indicated that two components of the secretory immune system, SC and intraepithelial $\operatorname{IgA}$, may disappear partly or completely from neoplastic epithelium. ${ }^{614}$ is-18 Carcinoembryonic antigen, on the other hand, which normally abounds only in the fetal intestinal epithelium, may appear in increased amounts in cancerous and inflammatory lesions of the large bowel. . $^{61719-21}$ Our present finding, indicating significantly more CEA and less SC and epithelial IgA in adenocarcinomas than in normal colonic mucosa, was, therefore, as expected. Moreover, the intermediate expression of these epithelial markers in the transitional mucosa confirmed our previous observations. $^{6}$

Carcinoembryonic antigen was fairly evenly distributed within individual tumours, as also reported by others. ${ }^{21}$ Conversely, small groups of epithelial elements positive for SC and IgA could occur in otherwise negative tumours. Such topical variability in the expression of SC and IgA, as well as differences between individual tumours, might in part depend on the growth pattern of the neoplastic epithelium. In gland-forming tumour elements, luminal transport of SC and dimeric IgA could presumably occur at a high rate and there would consequently be no excessive epithelial staining for these two markers. When no lumen was formed, on the other hand, expression of SC and uptake of dimeric IgA might result in cytoplasmic accumulation of both components and thereby disproportionately bright staining. Nevertheless, the overall positive relation previously noted ${ }^{6}$ between staining for both these markers in neoplastic epithelium and the degree of tumour differentiation was confirmed in our extended material.

Tumour metastases were commonly found to conform to the primary tumour with regard to SC and epithelial IgA (Rognum et al, unpublished observations), and measurements of secretory $\operatorname{IgA}$ in serum have previously been suggested as an adjunct in followups of SC- and IgA-positive carcinomas. ${ }^{22} 23$ It should be noted, however, that a weak negative correlation between tumour expression of SC (and IgA) and stage was indicated, and that a high proportion of the tumours were negative for both markers $(41 \%$ lacked SC and $53 \%$ lacked epithelial IgA).

Most authors have failed to find a clear relation between the degree of tumour differentiation and the expression of tumour CEA as evaluated by immunohistochemistry ${ }^{24}$ or by measurements of 
extractable antigen. ${ }^{2021}$ In our extended material there seemed to be, on the average, less CEA in well differentiated than in both moderately and poorly differentiated tumours; it should, nevertheless, be noted that the latter category contained four CEAnegative specimens. These results are in general agreement with those of Bordes et al ${ }^{26}$ but in contradiction to the claim of Denk et $a l^{27}$ that well differentiated tumours contain most CEA. However, only five moderately and two poorly differentiated adenocarcinomas were included in the latter study. We have, moreover, recently found that aneuploid tumours express significantly more CEA than near diploid ones, ${ }^{28}$ and heterogeneity of this variable probably explains some of the conflicting results in the literature.

In agreement with others, ${ }^{252629}$ we found that the transitional mucosa adjacent to tumours seemed to contain more CEA than normal, especially when hyperplastic epithelial elements were present. We also observed that increased expression of CEA was generally associated with decreased expression of SC and epithelial IgA in this zone, and that these alterations were related to the degree of tumour differentiation. In another $\operatorname{study}^{28}$ we have shown also that the DNA profile of the tumour is related to its influence on the expression of CEA, SC and epithelial IgA in the adjacent transitional mucosa. Filipe and Branfoot $^{30}$ have reported that a decreased sulphomucin-to-sialomucin proportion in the transitional mucosa was directly related to stage. We similarly found a relation between stage and decreased expression of $\mathrm{SC}$ and epithelial $\operatorname{IgA}$ in this zone. Both mucin- and immunohistochemistry of the transitional mucosa may hence contribute to elucidation of the malignant potential of large bowel carcinomas.

Pre- and postoperative measurements of plasma CEA concentrations may provide useful information in follow-ups of patients with large bowel carcinoma, ${ }^{2}$ but interpretation of data in clinicopathological terms is rendered difficult by numerous discrepant reports..$^{2431-34} \mathrm{We}$ found that the plasma CEA concentration tended to be higher in patients with localised moderately differentiated tumours than in those with well differentiated ones. On the whole, however, our study supported neither the opinion that poorly differentiated tumours are associated with particularly high plasma CEA concentrations ${ }^{24}{ }^{31}$ nor the suggestion that such tumours tend to release relatively little CEA. ${ }^{23}$ Our results are more in agreement with those of Bivins $e^{t} a^{32}$ who reported that plasma CEA concentrations were independent of tumour differentiation, and we have recently found that a tumour's capacity for CEA output is rather influenced by its DNA profile. ${ }^{28}$
Like Goslin et $a^{35}$ we have seen normal plasma CEA concentrations in patients with disseminated large bowel carcinomas that have been both poorly differentiated and negative for CEA staining; but we have also seen such a case associated with high plasma CEA concentration $(180 \mu \mathrm{g} / \mathrm{l})$. We do not agree, therefore, with the claim ${ }^{35}$ that patients with such tumours should be excluded from serial CEA measurements.

A positive correlation between stage and preoperative plasma CEA concentration has been reported by several authors ${ }^{263134}$ although not consistently so. ${ }^{32}$ Skewness in the distribution of stage within different patient groups may have influenced the results. ${ }^{32}$ In our material as a whole, this correlation was weak; but in the group of moderately differentiated tumours the plasma CEA concentrations were significantly higher in disseminated than in localised cases. As mentioned above, the DNA profile of the tumour seems to influence its output of CEA; the same variable is, moreover, apparently involved in the relation between stage and plasma CEA level. ${ }^{28}$ Results of CEA measurements thus have to be interpreted against a very complex biological background.

In conclusion, large bowel carcinomas show striking heterogeneity with regard to: (a) tumour expression of CEA, SC and epithelial IgA; (b) influence of tumour growth on the expression of the same epithelial markers by the adjacent transitional mucosa; and (c) capacity for release of CEA into plasma. Such heterogeneity can only to some extent be explained by taking into account the differentiation of tumour epithelium as judged by traditional histopathological criteria. However, both these criteria and the ways in which the functional variables have been assessed are necessarily static and extremely unsatisfactory in biological terms; only hints about meaningful correlations may hence be expected to emerge from such studies. Results of the present investigation indicated that further marker tracing in large bowel carcinomas is warranted to obtain a better functional basis for their classification. We plan to evaluate the prognostic significance of the variables included in this and another study ${ }^{28}$ by clinical follow-up of our patients.

This study was supported by the Norwegian Cancer Society and Oleand Rolf Nordberg's Legacy. The authors are grateful to Gunn Jamne, Vigdis Wendel and Elisabeth Falleth for skilled technical assistance.

\section{References}

' Dukes CE, Bussey HJR. The spread of rectal cancer and its effects on prognosis. Br J Cancer 1958;12:309-20. 
${ }^{2}$ National Institutes of Health Consensus Development Conference Statement. Carcinoembryonic antigen: its role as a marker in the management of cancer. Cancer Res 1981;41:2017-8.

${ }^{3}$ Burtin P. The carcinoembryonic antigen of the digestive system (CEA) and the antigens cross-reacting with it. Ann Immunol (Paris) 1978;129C: 185-98.

4 Brandtzaeg P. Transport models for secretory IgA and secretory IgM. Clin Exp Immunol 1981;44:221-32.

${ }^{5}$ Ejeckam GC, Huang SN, McCaughey WTE, Gold P. Immunohistopathologic study on carcinoembryonic antigen (CEA)-like material and immunoglobulin $\mathrm{A}$ in gastric malignancies. Cancer 1979;44:1606-14.

${ }^{6}$ Rognum TO, Brandtzaeg P, Ørjasaeter H, Elgjo K, Hognestad J. Immunohistochemical study of secretory component, secretory IgA and carcinoembryonic antigen in large bowel carcinomas. Pathol Res Pract 1980;170:126-45.

${ }^{7}$ Brandtzaeg P. Mucosal and glandular distribution of immunoglobulin components. Immunohistochemistry with a cold ethanol-fixation technique. Immunology 1974;26:1101-14.

- Stave R, Brandtzaeg P. Fluorescence staining pattern of gastric mucosa. A study with special reference to parietal cells. Scand J Gastroenterol 1977;12:885-91.

- Ashley JB. Evans' histological appearance of tumours. 3rd ed. Edinburgh: E \& S Livingstone, 1978.

${ }^{10} \emptyset$ rjasaeter H, Fosså S, Schjoeseth SA, Fjaestad K. Carcinoembryonic antigen in plasma of patients with carcinoma of the bladder/urethra. Cancer 1978;42:287-95.

"Stevens SS. On the theory of scales of measurement. Science 1946;103:677-80.

${ }^{12}$ Siegel S. Non-parametric statistics for the behavioral sciences. Tokyo: McGraw-Hill Kogakusha, 1956.

${ }^{13}$ Seifart W. Die therapeutische Situation beim Kolorektalen Karzinom. Arch Geschwulst forsch 1979;49:334-8.

14 Poger ME, Hirsch BR, Lamm ME. Synthesis of secretory component by colonic neoplasms. Am J Pathol 1976;82:327-38.

is Weisz-Carrington P, Poger ME, Lamm ME. Secretory immunoglobulins in colonic neoplasms. Am J Pathol 1976;85:303-16.

16 Green FHY, Whitehead S, Fox H. Abnormalities of the local immune system in intestinal neoplasia: A morphological study. J Pathol 1977:122:55-61.

" Rognum TO, Elgjo K, Fausa O, Brandtzaeg P. Immunohistochemical evaluation of carcinoembryonic antigen, secretory component, and epithelial $\operatorname{IgA}$ in ulcerative colitis with dysplasia Gut 1981;22.

is Rognum TO, Fausa O, Brandtzaeg P. Immunohistochemical evaluation of carcinoembryonic antigen, secretory component, and epithelial IgA in tubules and villous large bowel adenomas with different grades of dysplasia. Scand J Gastroenterol $1981 ; 17: 341$.

${ }^{19}$ Go VL, Spencer RJ, Ravry MJ, Shorter RG, Huizenga KA. Carcinoembryonic antigen (CEA) in malignant and inflammatory colon tissue. Gastroenterology 1973;64:734.

${ }^{20}$ Boyd CR, Bivins BA, Kashmiri R, Parker JC, Meeker WR. Plasma CEA, tumor CEA and tumor histology. J Surg Oncol 1976;8:507-12.

21 Wagener C, Müller-Wallraf R, Nisson S, Gröner J, Brener H Localization and concentration of carcinoembryonic antigen
(CEA) in gastrointestinal tumors: correlation with CEA levels in plasma. J Natl Cancer Inst 1981;67:538-47.

${ }^{22}$ LoGerfo $\mathrm{P}$. McLanahan S. Serum secretory IgA levels in patients with neoplastic disease J Surg Res 1976;20:481-4.

${ }^{23}$ Harris JP. South MA. Secretory component. A glandular epithelial cell marker. Am J Pathol 1981;105:47-53.

24 Pihl E, McNaughtan J, Ma J, Ward HA, Nairn RC. Immunohistological patterns of carcinoembryonic antigen in colorectal carcinoma. Correlation with staging and blood levels. Pathology 1980;12:7-13.

${ }^{25}$ O'Brian MJ, Zamcheck N. Burke B, Kirkham SE, Saravis CA, Gottlieb LS. Immunocytochemical localisation of carcinoembryonic antigen in benign and malignant colorectral tissues. Am J Clin Pathol 1981;75:283-90.

${ }^{26}$ Bordes M, Michiels R, Martin F. Detection by immunofluorescence of carcinoembryonic antigen in colonic carcinoma, other malignant or benign tumours, and non-cancerous tissues. Digestion 1973;9:106-15.

${ }^{27}$ Denk H, Tappeiner G, Eckersdorfer R, Holzner JH. Carcinoembryonic antigen (CEA) in gastrointestinal and extragastrointestinal tumors and its relationship to tumor-cell differentiation. Int J Cancer 1972;10:262-72.

${ }^{28}$ Rognum TO. Thorud E, Elgjo K, Brandtzaeg P, Orjasaeter H, Nygaard K. Large bowel carcinoma with different nuclear ploidy: Secretory component. IgA, and CEA in epithelial and $\mathrm{CEA}$ in plasma. $\mathrm{Br} J$ Cancer (in press).

29 Von Kleist S, Burtin P. Localisation cellulaire d'un antigene embryonnaire de tumeurs coliques humaines. Int $J$ Cancer 1969;4:874-9.

${ }^{30}$ Filipe MI, Branfoot AC. Abnormal patterns of mucus secretion in apparently normal mucosa of large intestine with carcinoma. Cancer 1974;34:282-90.

3' Zamcheck N, Doos WG, Prudente R, Lurie BB, Gottlieb LS. Prognostic factors in colon carcinoma. Correlation of serum carcinoembryonic antigen level and tumour histopathology. Hum Pathol 1975;6:31-45.

32 Bivins BA, Meeker WR, Griffin WO, Pellegrini J, Parker JC. Carcinoembryonic antigen (CEA) levels and tumour histology in colon cancer. J Surg Res 1975;18:257-61.

${ }^{33}$ Goslin R, Steele G, Macintyre J et al. The use of preoperative plasma CEA levels for the stratification of patients after curative resection of colorectal cancers. Ann Surg 1980;192:74751.

34 Lavin PT, Day J, Holyoke D, Mittelman A, Chu TM. A statistical evaluation of baseline and follow-up carcinoembryonic antigen in patients with resectable colorectal carcinoma. Cancer 1981;47:823-6.

${ }^{35}$ Goslin R, O'Brien MJ, Steele G, et al. Correlation of plasma CEA and CEA tissue staining in poorly differentiated colorectal cancer. Am J Med 1981;71:246-53.

Requests for reprints to: Dr TO Rognum, Histochemical Laboratory, Institute of Pathology, Rikshospitalet, Oslo 1, Norway. 